\title{
Aging Effects and Working Memory in Garden-Path Recovery
}

\author{
Hyunsoo Yoo ${ }^{1,2}$, Michael Walsh Dickey ${ }^{2,1}$ \\ 'University of Pittsburgh, Department of Communication Science \& Disorders, Pittsburgh; ${ }^{2}$ VA Pittsburgh Healthcare System, Pittsburgh, PA, USA
}

Purpose: The relationship between working memory (WM) and sentence comprehension (SC) has drawn many researchers' attention. The current study examined whether healthy older adults have difficulty comprehending potentially WM-demanding garden-path sentences involving syntactic ambiguities, and whether their comprehension is predicted by their working memory capacity or inhibitory control.

Methods: Older adults $(n=35$, ages 60-89) and younger adults $(n=36$, ages 19-33) completed two self-paced reading experiments and a battery of cognitive measures (working memory and inhibition tasks). Participants read the sentences containing a Minimal Attachment (MA) ambiguity in Experiment 1 and a Late Closure (LC) ambiguity in Experiment 2.

Results: The older adults' garden-path effect was larger than younger adults' in on-line measures. However, older adults exhibited higher off-line acceptability judgments for gardenpath sentences than younger adults, for both LC and MA sentences. Working memory predicted off-line performance of both younger and older groups in Experiment 2 (LC).

Conclusions: The results showed age-related differences in the comprehension of sentences with temporary syntactic ambiguities. Successful recovery from garden paths (revealed by off-line measures) was predicted by working memory. However, the source of age-related differences in real-time processing remains unclear. These results indicate that healthy aging can affect comprehension of challenging, syntactically ambiguous material.

Keywords: Sentence comprehension, Syntactic ambiguity, Garden-path sentences, Working memory, Inhibition

\section{INTRODUCTION}

Understanding syntactically ambiguous sentences has been a central research topic in the psycholinguistic literature. Sentences with syntactic ambiguities are often referred to as garden-path sentences [1], in that they lead comprehenders "down the garden path". These garden-path sentences cause readers or listeners to commit to an initial, incorrect interpretation of an ambiguous word or phrase, one that they must later revise after encountering additional information. Reanalysis happens when comprehenders read or hear that subsequent additional information, decide their initial interpretation was incorrect, and reinterpret the sentence. This reanalysis typically involves a change in the syntactic structure of the sentence and the syntactic role that the ambiguous word or phrase plays.

Frazier [2] proposed two syntactic parsing principles regarding garden-path sen-

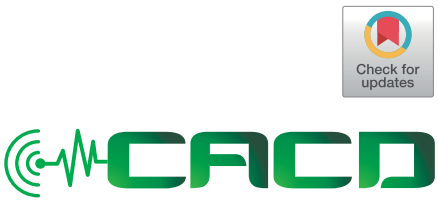

Received: June 30, 2017

Accepted: August 28, 2017

\section{Correspondence:}

Hyunsoo Yoo

6035 Forbes Tower, Communication Science \& Disorders, University of Pittsburgh, PA, USA

Tel: +1-412-926-8822

E-mail: hyunsooyoo@gmail.com

(C) 2017 The Korean Association of SpeechLanguage Pathologists

This is an Open Access article distributed under the terms of the Creative Commons Attribution Non-Commercial license (http:// creativecommons.org/licenses/by-nc/4.0/) which permits unrestricted non-commercial use, distribution, and reproduction in any medium, provided the original work is properly cited. 
tences that require reanalysis: Late Closure and Minimal Attachment. According to the first principle, Late Closure [2], comprehenders have a strong tendency to connect a word or phrase to the word that they read or heard most recently.

(1) a. While the man hunted the pheasant the deer ran into the woods.

b. While the man hunted the deer ran into the woods.

Comprehenders strongly tend to interpret "the deer" as the object of "hunted" initially in (1b), following the Late Closure principle. However, reanalysis of this initial analysis is required when the following verb, "ran", is processed. Encountering this verb forces comprehenders to revise their initial interpretation of "the deer" as the object of "hunted," and instead to treat it as the subject of "ran." Comprehenders experience processing difficulty when they must reanalyze the structure. This processing difficulty appears as increased reading or listening times for the verb "ran" in garden-path sentences like (1b) compared to non-garden-path control sentences like (1a) [3-8].

According to the second principle, Minimal Attachment [2], readers or listeners strongly prefer to make the simplest structure or interpretation of a phrase possible, given the grammatical constraints of their language.

(2) a. The editor played the tape and agreed the story was big.

b. The editor played the tape agreed the story was big.

Following Minimal Attachment, comprehenders strongly prefer to treat the first five words of (2a-b) as the structurally simplest and highly frequent structure, Subject-Verb-Object. As a result, "the editor" is initially interpreted as the subject of "played the tape." When comprehenders encounter the verb "agreed" in (2b), reanalysis for the structure is forced. They must change their interpretation to one in which "the editor" is in fact the person being played the tape, a low-frequency and more complex syntactic structure [2]. Comprehenders experience processing difficulty at the moment of reading or hearing the word "agreed" because of this reanalysis $[5,7,9]$.

The relationship between working memory (WM) and sentence comprehension (SC) has been a popular topic in SC studies since it is widely assumed that understanding sentences requires verbal WM resources (e.g., [10]). The relationship between WM and SC has been investigated in different ways, such as by examining individual differences, comparing people with high WM and people with low WM [11-14], or by comparing different populations such as persons with aphasia versus persons without aphasia [15-19] or younger versus elderly adults [20-23]. Many researchers have argued that WM limitations underlie SC differences across these populations, and may be responsible for SC impairments in aphasia (e.g., [14]). Understanding how WM and SC are related is therefore crucial for understanding SC in aphasia and in other conditions, such as healthy aging.

The focus of this paper is on age-related differences in SC (specifically syntactic ambiguity) in healthy aging, and their potential relationship to WM and inhibition. Data from healthy older adults often serves as the baseline for evaluating SC impairments among persons with aphasia. Understanding the nature of differences in SC performance due to healthy aging is therefore important for measuring SC performance in aphasia, and for evaluating the contribution of WM to SC impairments in aphasia. Although there is fairly consistent evidence that elderly adults have reduced WM capacity $[24,25]$, conflicting results have been found regarding whether elderly adults exhibit different SC behaviors from younger adults based on differences in WM [26-30]. These conflicting results include whether elderly adults show similar or poorer performance on SC compared to young adults, and how the different or similar results may be related to their WM capacity [23,31-35]. To date, there has been little effort to examine the possible sources of these conflicting results, or how they may be related to theoretical distinctions important to understanding the relationship between WM and SC more generally.

There are at least two theoretical distinctions from the SC and WM literature which are relevant to examining how SC is affected by healthy aging. The first of these issues is how specialized or domain-specific WM is: is WM a single resource, with one pool of memory resources being used for SC and other cognitively-demanding tasks (the SR theory of Just and Carpenter [13]), or does WM have a separate sentence interpretation resource, with dedicated resources used only for SC (the SSIR theory of Caplan and Waters [10]). The effects of aging on cognitive performance have often been assumed to be domain general rather than specific to language (e.g., [36,37]), so the question of whether older adults' SC performance is connected to their WM decline may speak to how specialized WM is. If older adults exhibit similar SC behavior to younger adults, then we may conclude that SC (in at least some aspects) does not require the sorts of WM resources which decline among older adults.

A related issue regarding the relationship between $\mathrm{WM}$ and SC is which aspects of SC rely most strongly on potentially limited WM resources. Caplan and Waters [10] proposed that different stages of processing may be differentially affected by 
limitations on WM. Interpretive processing is "the computation of a set of linguistic representations like lexical items, syntactic structures, intonational contours, thematic roles, focus and topic" ([10], p. 115), while post-interpretive processing is "remembering the contents of a sentence, using the meaning of a sentence to plan action, and reasoning on the basis of sentence meaning" ([10], p. 79). Interpretive processing is thus closely connected to real-time assembly of linguistic structure, and post-interpretive processing is additional cognitive processing based on the output of interpretive processing. These two different types of processing may draw differentially on WM resources, and may be measured using different methods. On-line measurements such as eye-tracking, self-paced reading/listening, or cross-modal lexical priming (CMLP) tap real-time processes relevant to interpretive processing, while off-line methods such as comprehension probes, grammaticality judgment, sentence-picture matching, sentence recall, or object manipulation tap processes more closely associated with post-interpretive processing. Regarding the relationship between $\mathrm{WM}$ and interpretive/post-interpretive processing, $\mathrm{WM}$ has been argued to be more closely related to post-interpretive processing [10, 28], particularly in healthy aging [20]. If this claim is correct, study-related factors like the choice of on-line versus off-line methodology may affect the likelihood of finding SC differences due to aging, as well as whether any aging-related SC differences are correlated with WM performance.

As evidence of the relationship between WM and Late Closure sentences, Christianson et al. [4] found that older adults' accuracy for the sentence final question was correlated with their WM, while younger adults' accuracy was not. These results provide support for the role of WM in successful reanalysis for Late Closure garden-path sentences, and they also show that reduced WM in older adults might affect the syntactic ambiguity resolution of Late Closure garden-path sentences.

As evidence of the relationship between WM and Minimal Attachment sentences, Kemper and colleagues [21] found that older adults with lower working memory made more regressions, and showed higher total reading times for Minimal Attachment garden-path sentences than younger adults did in the eye-tracking study. The results indicate that older adults experienced particular difficulty in reanalysis of Minimal Attachment garden-path sentences. In addition, Kemper et al. [21] found that working memory was as a mediator in the agerelated differences (reading times) for the reanalysis. That is, older adults with high working memory did not show difference in their regressions and total reading times from younger adults. The finding indicates the role of working memory in syntactic ambiguity resolution: how successfully older adults complete reanalysis of Minimal Attachment garden-path sentences.

Another aspect of cognition that is relevant to syntactic ambiguity resolution and which also declines with age is inhibitory function. Garden-path sentences require readers to abandon their initial interpretation when they encounter later words or phrases that are inconsistent with it, as part of reanalysis. This process requires successful inhibition of the previous interpretation. Novick et al. [38] present evidence from two adults with left-hemisphere pre-frontal damage and poor inhibitory function (as measured by Stroop-task performance, among other measures) illustrating the importance of intact inhibitory function for successful garden-path sentence comprehension. These adults exhibited good language comprehension in general but poor comprehension of syntactically ambiguous sentences. Consistent with this evidence, January and colleagues [39] found in an fMRI task that comprehension of garden-path sentences activated left-hemisphere areas which were also activated by Stroop task performance.

Inhibitory function also declines with age, like working memory [40], and some have argued that inhibitory deficits are responsible for apparent aging-related effects of working memory on language performance. Hasher and Zacks [36] argued that poor inhibitory control among older adults creates greater interference from task- and goal-irrelevant information, which interferes with successful retrieval of correct information from memory. A general decline in inhibitory function may therefore create special problems for comprehension of garden-path sentences. Such sentences require comprehenders to successfully inhibit the briefly tempting but ultimately incorrect garden-path interpretation during reanalysis. Failure to do so may cause older adults to misinterpret the sentence, based on the lingering garden-path interpretation. Failure to inhibit the garden-path structure may also create interference in memory, as comprehenders to try retrieve the misinterpreted phrases ("the deer" and "the editor played," in ( $\mathrm{b}-2 \mathrm{~b}$ ) above) and restructure them during reanalysis (viz. [36]). Such interference would lead to failed reanalysis, with comprehenders being unable to restructure the garden-path sentence correctly, possibly rejecting it as ungrammatical [6].

There are a number of studies investigating the relationship 
between aging, working memory, and sentence comprehension (e.g., [20,23,41]). Most have reported qualitatively similar on-line performances among older and younger adults. However, age-related differences appear primarily in off-line measures like post-sentential comprehension probes (e.g., [20, 34]). These off-line differences frequently correlate with measures of working memory such as span tasks (e.g., $[4,20])$ and are consistent with Caplan and Waters' assertion [10] that online interpretive processes are not affected by aging and do not rely on general working memory resources. There is continuing controversy regarding how sentence comprehension and language processing are supported by cognitive factors like working memory, inhibitory function, and speed of processing, and how these factors may be affected by aging (e.g., $[23,33])$.

However, there is a relative paucity of studies investigating the relationship between syntactic ambiguity resolution and aging specifically. Christianson et al. [4] examined older and younger adults' comprehension of garden-path sentences like (la-b), involving Late Closure syntactic ambiguities. They asked both groups end-of-sentence comprehension questions about the garden-path interpretation ("Did the man hunt the deer?"), which should be inhibited during reanalysis. They did not find a difference between older and younger adults in how often the two groups correctly answered the comprehension questions, indicating that the two groups were similarly successful in inhibiting the garden-path interpretation. This similarity suggests that older adults' comprehension of Late Closure garden-path sentences is similar to younger adults', and that older adults did not exhibit reduced inhibitory function (contra [36]). However, they did not directly measure either group's inhibitory function, so this conclusion must be treated with caution.

The current study examined older and younger adults' comprehension of both Late Closure and Minimal Attachment garden-path sentences like (1-2). To date, no study has directly compared older and younger adults' comprehension of both these types of garden-path sentences in the same study. The primary goals of the study were to determine whether older adults have more difficulty in comprehending garden-path sentences than younger adults, and to determine whether working memory or inhibitory function predicted any age-related differences. Some previous findings suggest that older adults are as successful in reanalyzing garden-path sentences as younger adults (e.g., [4]), while other findings indicate that reanalysis may be more difficult for older adults (e.g., [21]). In addition, some previous work has claimed that working memory is responsible for age-related differences in sentence comprehension [13], while others have argued that changes in inhibitory function are responsible for age-related language comprehension effects [36], or may negatively impact garden-path sentence comprehension specifically [38]. A secondary goal of the study was to examine whether age-related differences in garden-path sentence comprehension appear in off-line or on-line measures. Some have argued that age-related sentence comprehension differences appear primarily in off-line measures $[10,20]$, while other argue that such differences should appear in both on- and off-line measures, especially if they are related to changes in working memory [13].

\section{METHODS, EXPERIMENTS 1-2}

\section{Participants}

A total of 71 subjects ( 36 younger adults and 35 older adults) participated in this study. All participants met the following selection criteria: (a) they were native American English speakers; (b) had at least 12 years of education; (c) had normal or corrected-to-normal vision; (d) passed a simplified reading screening; (e) performed within normal limits on the language recall task of the Assessment Battery of Communication in Dementia (ABCD) [42] with a ratio (the delayed recall/immediate recall $\times 100$ ) greater than 0.70 ; (f) had normal visual memory by Rey-Osterrieth Complex Figure [43]; (g) passed a bilateral pure-tone hearing screen at $40 \mathrm{~dB}$; (h) scored 27 or above on the Mini-Mental State Examination (MMSE; [44]). Participants in the younger group ranged in age from 18 to 35 years, and all members of the older group were over 60 years of age. Table 1 provides descriptive and screening measures for all participants for this study.

\section{Materials}

Cognitive measures: Sentence-span [45], Subtract 2 span [46], Forward- and Backward-digit span tasks [17], and the Flankers task [47] were administered to test working memory and inhibitory function. These tests are described in more detail in the Procedures below.

Language processing measures: Participants read 24 Late Closure sentences like (3a-b) in Experiment 1, and 20 Minimal Attachment sentences like (4a-b) Experiment 2.

(3) a. While | the man | hunted | the pheasant | the deer | ran | into| the woods. 
Table 1. Descriptive information and screening test results for participants

\begin{tabular}{|c|c|c|c|c|c|c|c|c|c|c|c|}
\hline \multirow[t]{2}{*}{ Age groups } & \multirow{2}{*}{$\begin{array}{c}\begin{array}{c}\text { Number of } \\
\text { subjects }\end{array} \\
\mathrm{N}\end{array}$} & \multicolumn{2}{|c|}{ Age } & \multicolumn{2}{|c|}{ Years of education } & \multicolumn{2}{|c|}{$\begin{array}{c}\text { MMSE } \\
(\operatorname{Max}=30)\end{array}$} & \multicolumn{2}{|c|}{$\begin{array}{l}\text { Plausibility } \\
\text { (Max=20) }\end{array}$} & \multicolumn{2}{|c|}{$\begin{array}{l}\text { ABCD retelling } \\
(\operatorname{Max}=100)\end{array}$} \\
\hline & & $M$ & SD & $M$ & SD & $M$ & SD & $M$ & SD & $M$ & SD \\
\hline Younger adults & 36 & 23 & 2.83 & 15.5 & 0.71 & 29.5 & 0.5 & 19.5 & 0.5 & 98 & 6 \\
\hline Older adults & 35 & 70 & 5.66 & 15.5 & 4.95 & 29.5 & 0.5 & 17 & 3 & 100 & 0 \\
\hline
\end{tabular}

b. While $\mid$ the man $\mid$ hunted $\mid$ the deer $\mid$ ran $\mid$ into $\mid$ the woods. (4) a. The editor | played | the tape | and | agreed | the story | was big.

b. The editor $\mid$ played $\mid$ the tape $\mid$ agreed $\mid$ the story $\mid$ was big.

The ' $\mid$ ' indicates presentation regions for the self-paced reading task. The Late Closure items were adapted from the stimuli in Christianson et al. [3] Experiment 1, while the Minimal Attachment items were adapted from the stimuli in Ferreira and Clifton's [5] Experiment 2.

\section{Procedures}

Cognitive measures: Sentence-span, forward- and backwarddigit span tasks were presented using the E-Prime 1.0 software program [48], and the Subtract 2 span task was presented orally by a researcher. In the sentence-span test, participants had to read sentences displayed on the computer screen and were instructed to remember the final word of each sentence. They were asked to recall the final words after making a grammaticality judgment regarding the sentences. For the forwardand backward-digit span tasks, the participants listened to a series of digits and had to point to those digits in order or in reverse order from 9 digits displayed on the computer screen. For the Subtract 2 span test, participants listened to a series of digits and had to repeat those digits out loud and in order after subtracting 2 from each digit. In the Flankers task, the five arrows with congruent (i.e. $\rightarrow \rightarrow \rightarrow \rightarrow \rightarrow$ and $\leftarrow \leftarrow \leftarrow<\leftarrow$ ) and incongruent (i.e. $\leftarrow \leftarrow \rightarrow \leftarrow$ ) conditions were presented using EPrime. The participants had to pay attention to the direction of the middle arrow and ignore the direction of the flanking arrows, then press right or left button on the button box based on the direction of the middle arrow.

Language processing stimuli: Participants read in a selfpaced segment-by-segment reading task without cumulative display. Participants were presented with a row of dashes as a preview and pressed the space bar to reveal each segment of the sentence. After reading each sentence, participants responded to a speeded grammaticality judgment task prompt of "Acceptable?" and responded by pressing one of two but- tons marked "YES" or "NO". The percentage (\%) of YES responses was computed in order to get the "acceptability rate" for analysis.

\section{Data analysis}

Cognitive measures: Based on an exploratory factor analysis [49], data from the working memory tasks were combined to form two working memory composite scores: WM1 and $\mathrm{WM} 2$. The composite scores for WM1 were derived from the Subtract 2 span and the Forward- and Backward-digit span tasks (percentage correct scores), because the factor analysis revealed that these three tasks loaded onto a single factor. In contrast, the WM2 was based on the Sentence-span task only (the number of recalled words), since performance on this task loaded onto a different factor from the other memory tasks. The Flankers task loaded onto a third, separate factor, one presumably related to inhibition/inhibitory function.

Language processing measures: For data analysis of on-line performance, reading times for the disambiguating region of each sentence requiring reanalysis were collected and analyzed. The disambiguating region is underlined in (3-4) above. The reading times for the following two regions immediately after the disambiguating region were also measured. Longer reading times in the garden-path condition ( $3 \mathrm{~b}, 4 \mathrm{~b})$ compared to the non-garden path condition is evidence of elevated processing related to reanalysis. For data analysis of off-line performance, the acceptability rate (percentages YES responses) for the garden-path condition (3b, 4b) was computed for each sentence. A higher acceptability rate for the garden-path condition means more successful reanalysis for the garden-path sentence [6].

\section{RESULTS}

\section{Cognitive measures}

The older age group performed significantly worse on the WM1 and WM2 composite scores and on the Flankers task than the younger group (Mann-Whitney $\mathrm{U}, \mathrm{WM1}: \mathrm{z}=-6.21$, 
$p<0.001$; WM2: $\mathrm{z}=-5.30, p<0.001$; Flankers: $\mathrm{z}=-2.40, p<0.05$ ). Therefore, these results support that the claim that older adults have more limited working memory [14] and poorer inhibitory control [36] than younger adults. The results for the cognitive measures are shown in Table 2.

\section{Language processing measures}

\section{Experiment1: Late closure: On-line results}

Older adults were significantly slower than younger adults in the garden-path condition (3b) at the disambiguating verb (Mann-Whitney $\mathrm{U}: \mathrm{z}=-4.669, p<0.001$ ) and the following two segments $(\mathrm{z}=-4.462, \mathrm{z}=-4.485$, both $p<0.001)$ (see Figure 1 ). Also, reading times were significantly higher for the gardenpath condition (3b) than for the non-garden-path condition (3a), for both groups. For the younger group, this pattern held at the disambiguating segment (Wilcoxon Signed Ranks, $\mathrm{z}=$ -4.415, $p<0.001)$ and the following segment $(\mathrm{z}=-4.462$, $p<0.001)$, but not the segment thereafter $(\mathrm{z}=-0.770, p>0.05)$. For the older group, this pattern held at the disambiguating segment $(\mathrm{z}=-4.472, p<0.001)$ but not at the following two seg- ments $(\mathrm{z}=-1.736$ and $\mathrm{z}=-1.065, p>0.05)$. Most importantly, there were between-group differences in the magnitude of the reading-time difference between the garden-path and nongarden path conditions (Mann-Whitney $\mathrm{U}, \mathrm{z}=-2.289, p<0.05$ ). This difference in the magnitude of the garden-path effect appeared only at the disambiguating segment.

\section{Experiment2: Minimal attachment: On-line results}

Older adults were significantly slower than younger adults in the garden-path condition (4b) at disambiguating verb (Mann Whitney U: $\mathrm{z}=-4.014, p<0.001)$ and the following two segments $(\mathrm{z}=-3.818, \mathrm{z}=-5.095$, both $p<0.001)$ (see Figure 2). Also, reading times were significantly higher for the gardenpath condition (4b) than for the non-garden-path condition (4a), for both groups. For the younger group, this pattern held at the disambiguating segment (Wilcoxon Signed Ranks, $\mathrm{z}=$ -3.723, $p<0.001)$ and the following segment $(\mathrm{z}=-3.488, p<$ $0.001)$, but not the segment thereafter $(\mathrm{z}=-1.288, p>0.05)$. For the older group, this pattern held at the disambiguating segment and the following two segments $(\mathrm{z}=-4.799, \mathrm{z}=-4.799$,

Table 2. Working memory (WM) \& inhibition tasks results

\begin{tabular}{|c|c|c|c|c|c|c|c|c|c|c|c|c|}
\hline \multirow[t]{2}{*}{ Age groups } & \multicolumn{2}{|c|}{$\begin{array}{c}\text { Sentence span } \\
\text { task (WM2) } \\
\text { (\% of recalled } \\
\text { words) }\end{array}$} & \multicolumn{2}{|c|}{$\begin{array}{l}\text { Digit span forward } \\
\text { (\% of correct } \\
\text { response) }\end{array}$} & \multicolumn{2}{|c|}{$\begin{array}{l}\text { Digit span } \\
\text { backward } \\
\text { (\% of correct } \\
\text { response) }\end{array}$} & \multicolumn{2}{|c|}{$\begin{array}{l}\text { Subtract-2-span } \\
\text { (\% of correct } \\
\text { response) }\end{array}$} & \multicolumn{2}{|c|}{$\begin{array}{l}\text { Composite score } \\
\text { (WM 1): Digit span } \\
\text { tasks + Subtract } 2\end{array}$} & \multicolumn{2}{|c|}{$\begin{array}{c}\text { Flanker } \\
\text { Reaction time (ms) } \\
\text { of Incongruent } \\
\text { minus congruent } \\
\text { condition }\end{array}$} \\
\hline & $M$ & SD & $M$ & SD & $M$ & SD & $M$ & SD & $M$ & SD & $M$ & SD \\
\hline Younger adults & 55.08 & 4.5 & 65.39 & 6.5 & 56.64 & 0.97 & 58.37 & 2 & 20.89 & 3 & 57.13 & 23.67 \\
\hline Older adults & 38.61 & 9.5 & 57 & 0 & 42.94 & 19.54 & 42.67 & 7 & 15.11 & 2 & 70.78 & 22.17 \\
\hline
\end{tabular}

Reading time of $\mathrm{LC}$

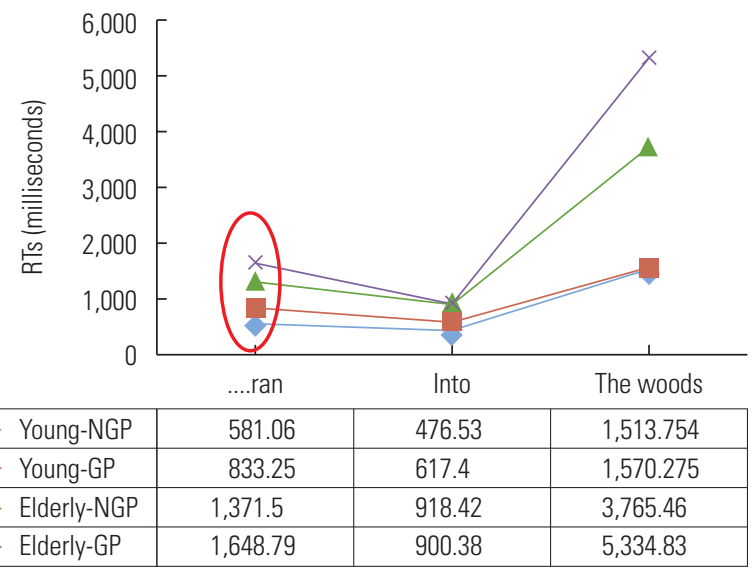

Figure 1. Reading time for disambiguating region for LC sentences (Experiment 1).

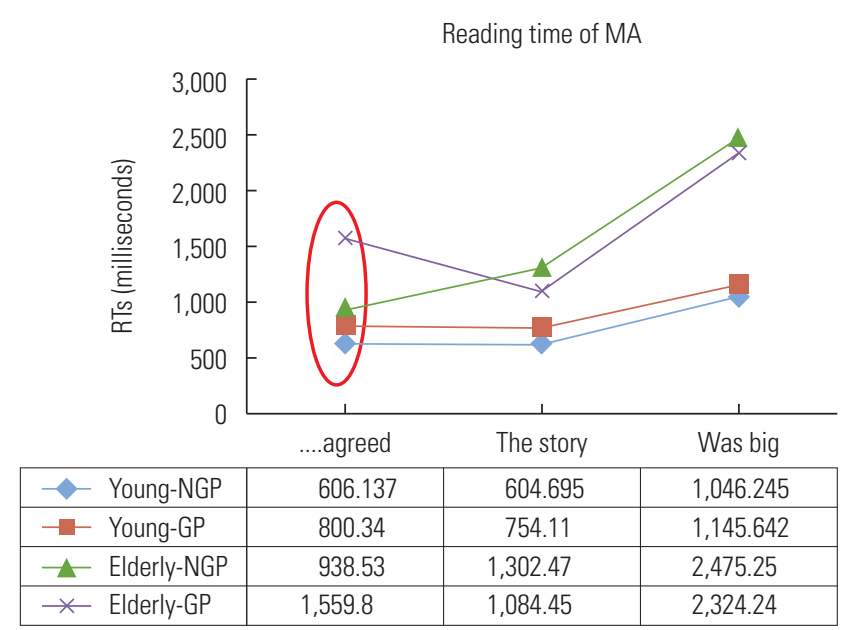

Figure 2. Reading time for disambiguating region for MA sentences (Experiment 2). 


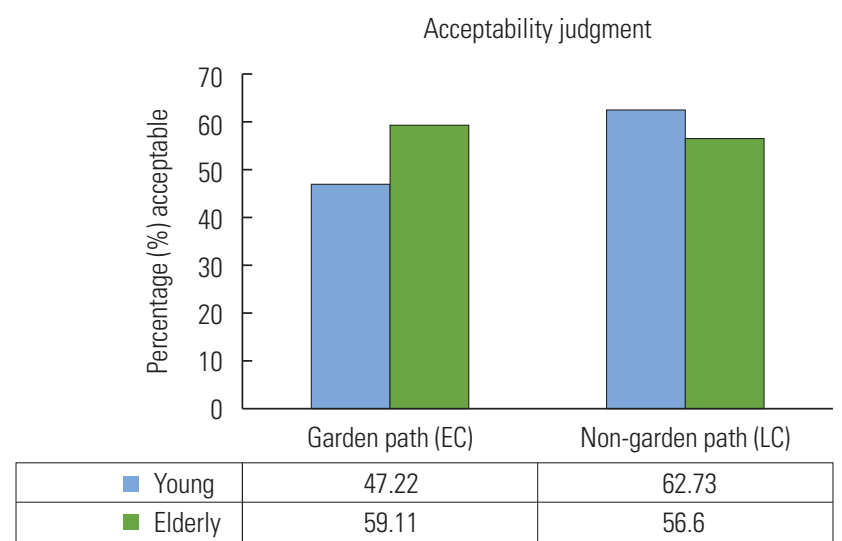

Figure 3. Acceptability rate (\% Yes responses) for LC sentences (Experiment 1).

$\mathrm{z}=-4.799$, all $p<0.001)$. Most importantly, there were between-group differences in the magnitude of the reading-time difference between the garden-path and non-garden path conditions (Mann-Whitney $\mathrm{U}, \mathrm{z}=-2.208, p<0.05$ ). This difference in the magnitude of the garden-path effect appeared only at the disambiguating segment.

In summary, older and younger adults exhibited gardenpath effects for both Late Closure and Minimal Attachment garden-path sentences, and the size of the garden-path effect was significantly larger for older adults. This finding is consistent with previous findings suggesting that older adults experienced more difficulty in reanalyzing Late Closure [50] and Minimal Attachment [21] garden-path sentences.

\section{Experiment1: Late closure: Off-line results}

As shown in Figure 3, mean differences in the acceptability rate for garden-path versus non-garden-path sentences were found for younger but not older adults. In the younger group, the garden-path condition (3b) was judged acceptable significantly less often than the non-garden path condition (3a) (Wilcoxon Signed Ranks, $\mathrm{z}=-3.079, p<0.05$ ). However, there was no difference between garden-path and non-garden path conditions for the older group $(\mathrm{z}=-0.618, p>0.05)$. Also, older adults judged Late Closure garden-path sentences unacceptable significantly less often than younger adults did (MannWhitney $U, z=-1.630, p>0.05)$. This finding suggests that older adults were more successful in reanalyzing Late Closure garden-path sentences as a group than younger adults were.

\section{Experiment2: Minimal attachment: Off-line results}

As shown in Figure 4, mean differences in the acceptability rate for garden-path versus non-garden-path sentences were

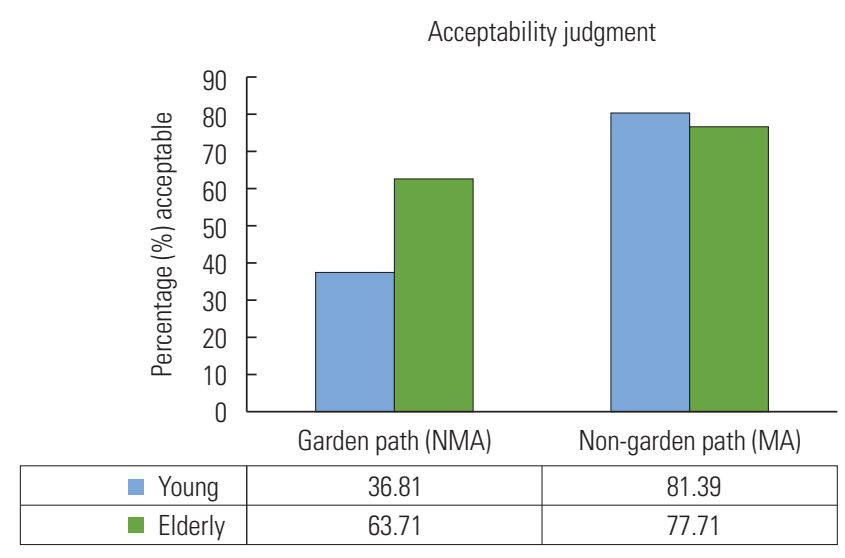

Figure 4. Acceptability rate (\% Yes responses) for MA sentences (Experiment 2).

found for both younger and older adults. The garden-path condition (4b) was judged acceptable significantly less often than the non-garden path condition (4a) by the younger group (Wilcoxon Signed Ranks, $\mathrm{z}=-5.086, p<0.001$ ) and the older group ( $\mathrm{z}=-3.234, p<0.05)$. Furthermore, older adults judged Minimal Attachment garden-path sentences unacceptable significantly less often than younger adults did (Mann-Whitney $\mathrm{U}, \mathrm{z}=-4.542, p<0.01$ ). This finding suggests that older adults were more successful in reanalyzing Minimal Attachment garden-path sentences than younger adults were.

In summary, both age groups judged garden-path conditions acceptable less often than non-garden path conditions. This is in line with previous findings showing that gardenpath sentences are judged unacceptable more often than non-garden-path sentences, because comprehenders have failed to successful reanalyze their structure [6]. Also, older adults judged garden-path sentences unacceptable less often than younger adults. This finding suggests that older adults had greater success in reanalyzing garden-path sentences than younger adults.

\section{Regression analyses: On-line results}

Bootstrapping regression analyses [51] examined the relationship between the on-line results (the magnitude of the garden-path effect) and the three cognitive factors identified in the exploratory factor analysis (Inhibition: Flankers score; Working memory: WM1 composite score, WM2 score). Each participant's served as a data point in the regression model. The size of the garden-path effect in reading times was based on a reading time difference score, derived by subtracting the mean reading times for the non-garden-path condition from the garden-path condition for Experiments 1 and 2 (Late Clo- 
sure and Minimal Attachment cases, respectively). The BCa Percentile intervals at $95 \%$ were calculated using Bootstrapping procedures, and they served as benchmarks for evaluating whether the cognitive measures predicted the gardenpath difference scores.

Interestingly, there was no prediction of the garden-path difference for Late Closure (LC) sentences (Experiment 1) or Minimal Attachment (MA) sentences (Experiment 2) by any of the cognitive factors, individually or in combination, for either group. This finding suggests that the source of the differences in on-line processing (on-line garden-path effect) is not working memory or inhibition, and implies that some other factors might be related to these on-line differences.

\section{Regression analyses: Off-line results}

Bootstrapping regression analyses [51] also examined the relationship between off-line measures (acceptability judgment task) and the three cognitive factors. The acceptability rate (percentage of YES responses) from the garden-path condition for LC stimuli (Experiment 1) and MA stimuli (Experiment 2) was used for this analysis. The $B C a$ Percentile intervals at $95 \%$ were once again calculated using Bootstrapping technqiues. In the younger group, the acceptability rate of LC garden-path condition was predicted by WM2 (sentence span task), BCa Percentile Intervals $=-0.0217,95 \% \mathrm{CI}=(-0.0247$, -0.0033), $R$ square $=0.0071$. In the older group, the acceptability rate of the LC garden-path condition was predicted by $\mathrm{WM1}$ (composite scores), BCa Percentile Intervals $=-0.0176$, $95 \% \mathrm{CI}=(-0.0292,-0.0059)$ and WM2 (sentence span task), BCa Percentile Intervals $=0.0080,95 \% \mathrm{CI}=(0.0018,0.0157), R$ square $=0.1857$. Acceptability judgment rates for MA gardenpath conditions were not predicted by any of the cognitive factors, individually or in combination, for either group.

In summary, the results from the regression analysis showed no predictions of on-line performance by any of the cognitive

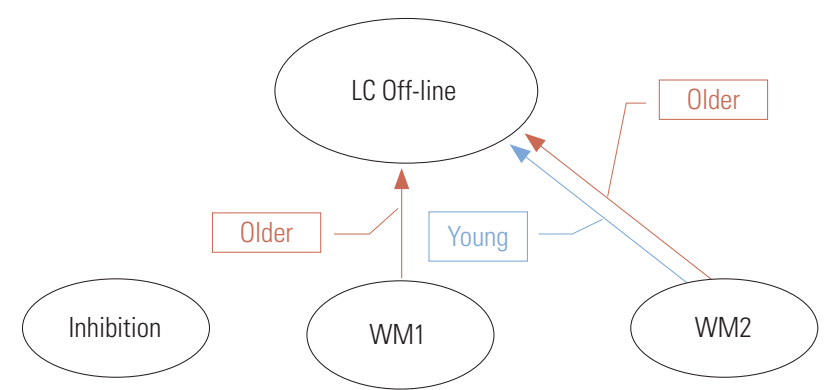

Figure 5. Predictions from bootstrap regression analyses. factors, for either group. However, the off-line results showed several predictions of LC performance. All predictions of acceptability judgments were by factors related to working memory, both for younger (WM2) and older groups (WM1 and WM2) (see Figure 5). These results showing that differences in cognitive measures such as working memory and inhibitory control affect only off-line measurement are consistent with the results (regarding working memory) from DeDe et al. [20]. Furthermore, the finding that working memory performance predicts off-line judgments for Late Closure sentences is consistent with Christianson et al.'s [4] finding showing that older adults' comprehension question accuracy for Late Closure sentences was predicted by their working-memory performance.

\section{DISCUSSION}

The primary goals of the current study were to investigate whether older adults exhibit difficulty in recovering from syntactic garden paths, and to investigate whether this difficulty is predicted by cognitive factors, working memory and inhibition. A secondary goal was to examine whether any age-related differences in garden-path sentence comprehension appeared in off-line measures, on-line measures, or both.

The primary results for this study are summarized in Tables 3 and 4. As shown in Table 3, there were on-line garden-path effects for both older and younger adults. There were also aging effects in the reading times for the disambiguating segment in both Experiment 1 (Late Closure garden-path sen-

Table 3. Patterns of results from on-line and off-line measurements

\begin{tabular}{|c|c|c|c|c|c|c|}
\hline & \multicolumn{3}{|c|}{ On-line } & \multicolumn{3}{|c|}{ Off-line } \\
\hline & \multicolumn{2}{|c|}{$\begin{array}{c}\text { Garden-path } \\
\text { effect }\end{array}$} & \multirow{2}{*}{$\begin{array}{c}\begin{array}{c}\text { Aging } \\
\text { effect }\end{array} \\
\text { Yes }\end{array}$} & \multicolumn{2}{|c|}{$\begin{array}{c}\text { Garden-path } \\
\text { effect }\end{array}$} & \multirow{2}{*}{$\begin{array}{c}\begin{array}{c}\text { Aging } \\
\text { effect }\end{array} \\
\text { No }\end{array}$} \\
\hline LC & Younger & Yes & & Younger & Yes & \\
\hline & Older & Yes & & Older & No & \\
\hline \multirow[t]{2}{*}{ MA } & Younger & Yes & Yes & Younger & Yes & No \\
\hline & Older & Yes & & Older & Yes & \\
\hline
\end{tabular}

Table 4. Patterns of predictions in regression analyses

\begin{tabular}{llllc}
\hline \multicolumn{3}{c}{ On-line } & \multicolumn{2}{c}{ Off-line } \\
\hline LC & Younger & No & Younger & Yes (by WM2) \\
& Older & No & Older & Yes (by WM1 \& WM2) \\
MA & Younger & No & Younger & No \\
& Older & No & Older & No \\
\hline
\end{tabular}


tences, LC) and Experiment 2 (Minimal Attachment gardenpath sentences, MA). In both cases, older adults showed a larger garden-path effect, indicating greater processing difficulty when they were forced to reanalyze their initial interpretation of the sentence. In the off-line measures, a different pattern appeared: older adults appeared to be more successful in revising both LC and MA garden-path sentences than younger adults. However, as shown in Table 4, there was no prediction of on-line performance by working memory and inhibitory control for either group: the observed differences between older and younger adults do not appear to be explained by the differences in working memory or inhibitory function between the groups. Nevertheless, there was a prediction of off-line performance for Late Closure sentences by working memory factors, for both groups. As noted above, this is a direct replication of Christianson et al.'s [4] finding that comprehension question accuracy (reflecting successful reanalysis) was correlated with working memory scores, found here not only for older but also for younger adults.

With respect to the primary goals of the study, two different pictures emerge. First, there were significant aging effects (differences in size of garden-path effect between the two age groups) in on-line measures for Experiment 1 and Experiment 2. This is consistent with previous findings suggesting that older adults may have particular difficulty with reanalysis, going back and re-structuring words and phrases once they have discovered their initial interpretation is incorrect (e.g., $[21,50])$. It is not consistent with the view of Caplan and Waters [10] and other similar studies' results $[20,28,31,52]$, which suggest that on-line processing is not affected by aging. One possible explanation of the differences between the current study and previous studies which failed to find aging effects in on-line measures is that the previous studies used different types of sentence stimuli, such as object relative clauses. These sentences do not require reanalysis, which appears to impose particular burdens for older adults.

Second, even though there are aging effects in this study, they do not appear to be mediated by working memory [13] or inhibitory function [36]. There was no prediction of the online effects by either memory- or inhibition-related cognitive factors, even though these factors did predict off-line performance (a finding we will return to below). This finding is not explained by approaches that account for age-related changes in sentence comprehension in terms of working memory (e.g., [13]) or inhibitory function [36]. It also indicates that other factors must underlie the age-related differences in the real- time processing of garden-path sentences. One possible candidate is speed of processing, which also declines with age and which other work has indicated may play a role in language comprehension [23,40,41]. Additional work is needed to determine whether speed of processing is what is responsible for the age-related differences in garden-path reanalysis found here.

With respect to the secondary goal of the study, two pictures again emerge. First, there were clear aging effects in the online measures for Experiment 1 and Experiment 2. As noted above, this is not consistent with previous results suggesting that on-line processing is immune to aging ([20,28,31,52]; viz., [10]). Second, older adults exhibited higher acceptability rates than younger adults, meaning that older adults more successfully revised the initial garden-path interpretation than younger adults did. The results are not consistent with Christianson et al's [4] finding that older adults showed lower accuracy than younger adults in comprehension probes, but did not show a particular disadvantage for garden-path sentences. One possible explanation for this finding is that more time spent in on-line processing may lead to more successful reanalysis among older adults. Consistent with this is Caplan et al's [41] suggestion that high working memory readers may spend more time at high points of complexity and consequently process the sentence more deeply, resulting in more successful off-line comprehension. Another possibility is that the type of off-line task may have affected the depth of understanding of sentences. The off-line task for the current study was an "acceptability task" only requiring shallow processing on the sentences while the off-line task in Christianson et al's [4] study was comprehension probes asking "who did what to whom" which requires a deep level of understanding of a sentence. Thus, these different demands in each task may affect the direction of the off-line results.

One other finding from the off-line results which merits discussion is the performance of older adults in Experiment 1, with Late Closure sentences. Acceptability rates were higher for non-garden-path than garden-path sentences for both age groups in Experiment 2 (Minimal Attachment), but only for young adults in Experiment 1 (Late Closure). Older adults did not show significant differences between garden-path and non-garden-path sentences in Experiment 1. The exact source of this difference is unclear, but it is worth noting that the acceptability rate for the non-garden path sentence in Experiment 1 ("While the man hunted the pheasant the deer ran into the woods") was unusually low compared to the typical 
acceptability rate of $80 \%$ for non-garden-path sentences in previous studies $[6,53]$. This pattern may indicate that both age groups found the non-garden-path sentence relatively unacceptable, perhaps because they were garden-pathed by the combination of "the pheasant the deer." Ferreira and Henderson $[6,53]$ report that some garden-path sentences (those with a longer distance between the head of misanalysed phrase and the disambiguating region) had an acceptability rate similar to the non-garden-path sentences of Experiment 1 (around $60 \%$ ). It could be that the combination of "the pheasant the deer" caused a garden-path, perhaps being briefly misinterpreted as part of a larger conjunction ("While the man hunted the pheasant the deer and the squirrel ..."). Further work is necessary to test this possibility.

\section{CONCLUSIONS}

The current study examined how healthy older and younger adults comprehend challenging garden-path sentences, which involve syntactic ambiguities. Such sentences have been extensively studied in the psycholinguistic literature but have received little attention in the communicative impairment literature, despite evidence that neurogenically impaired adults may have difficulty understanding sentences requiring reanalysis $[38,54]$. The findings here suggest that healthy aging can affect how adults understand these sentences, with greater processing loads when they must reanalyze. However, this greater reanalysis difficulty appears unlikely to be due to age-related changes in working memory or inhibitory function. Subsequent work will need to address the source of these age-related changes, as well as to take into consideration the effects of healthy aging when testing how language-impaired adults deal with ambiguous material such as garden-path sentences.

\section{ACKNOWLEDGMENTS}

This research was supported by an SHRS Research Development Fund award to Hyunsoo Yoo, by a University of Pittsburgh Central Research Development Fund grant to Michael Walsh Dickey, and by grant number UL1TR000005 to the Clinical and Translational Science Institute of the University of Pittsburgh. This research is the result of work supported with resources and the use of facilities at the VA Pittsburgh Healthcare System. The authors are grateful to audiences at the 2011 CUNY Conference on Human Sentence Processing and the 41st Clinical Aphasiology Conference for helpful discussion, and to Will Hula for assistance with factor analysis. The authors are particularly grateful to the residents of the Benedictine Center of Pittsburgh, PA, who participated in this study.

\section{REFERENCES}

1. Bever TG. The cognitive basis for linguistic structures. In: J.R. Hayes, Editor, Cognition and the development of language, New York:Wiley;1970;279-362.

2. Frazier L. On comprehending sentences: Syntactic parsing strategies. Bloomington, IN: Indiana University Linguistics Club;1979.

3. Christianson K, Hollingworth A, Halliwell JF, Ferreira F. Thematic roles assigned along the garden path linger. Cognitive Psychology. 2001;42(4):368-407.

4. Christianson K, Williams CC, Zacks RT, Ferreira F. Younger and Older Adults' "Good-Enough" Interpretations of Garden-Path Sentences. Discourse Processes, 2006;42(2):205-2388.

5. Ferreira F, Clifton C. The independence of syntactic processing. Journal of Memory and Language. 1986;25:348-368.

6. Ferreira F, Henderson JM. Recovery from misanalyses of gardenpath sentences. Journal of Memory and Language. 1991;25:725745.

7. Frazier L, Rayner K. Making and correcting errors during sentence comprehension: eye movements in the analysis of structurally ambiguous sentences. Cognitive Psychology. 1982;14:178-210.

8. Pickering MJ, Traxler MJ. Plausibility and recover from gardenpaths: An eye-tracking study. Journal of Experimental Psychology: Learning, Memory, and Cognition. 1998;24:940-961.

9. Trueswell JC, Sekerina I, Hill NM, Logrip ML. The kindergartenpath effect: studying on-line sentence processing in young children. Cognition. 1999;73:89-13

10. Caplan D, Waters G. Verbal working memory capacity and language comprehension. Behavioral and Brain Science. 1999;22: 114-126.

11. Daneman M, Carpenter P. Individual differences in working memory and reading. Journal of Verbal Learning and Verbal Behavior. 1980;19:450-466.

12. King J, Just MA. Individual differences in syntactic processing: The role of working memory. Journal of Memory and Language. 1991;30:580-602.

13. Just MA, Carpenter PA. A capacity theory of comprehension: Individual differences in working memory. Psychological Review. 1992;99:122-149.

14. Carpenter PA, Miyake A, Just MA. Working memory constraints in comprehension: Evidence from individual difference, aphasia and aging. In M. A. Gernsbacher (Ed.), Handbook of psycholinguistics. New York: Academic Press. 1994;1075-1122.

15. Caspari I, Parkinson S, LaPointe L, Katz R. Working memory and aphasia. Brain and Cognition. 1998;37:205-223.

16. Friedmann N, Gvion A. Sentence comprehension and working 
memory limitation in aphasia: A dissociation between semanticsyntactic and phonological reactivation. Brain and Language. 2003;86:23-39.

17. Martin N, Kohen FP, Kalinyak-Fliszar M. A processing approach to the assessment of language and verbal short-term memory abilities in aphasia. Clinical Aphasiology Conference; Charleston, SC. 2010.

18. Wright HH, Shisler R. Working memory in aphasia: Theory, measures, and clinical implications. American Journal of Speech-Language Pathology. 2005;14:107-118.

19. Wright HH, Downey RA, Gravier M, Love T, Shapiro LP. Processing distinct linguistic information types in working memory in aphasia. Aphasiology. 2007;21:802-813.

20. DeDe G, Caplan D, Kemtes K, Waters G. The relationship between age, verbal working memory and language comprehension. Psychology and Aging. 2004;19:601-616.

21. Kemper S, Crow A, Kemtes K. Eye fixation patterns of high and low span young and older adults: Down the garden path and back again. Psychology and Aging. 2004;19:157-170.

22. Kemper S, Herman R. Age differences in memory load interference effects in syntactic processing. Journal of Gerontology: Psychological Sciences. 2006;61B:327-332.

23. Kwong See ST, Ryan EB. Cognitive mediation of adult age differences in language performance. Psychology and Aging. 1995;10: 458-468.

24. Wingfield A, Stine EL, Lahar CJ, Aberdeen JS. Does the capacity of working memory change with age? Experimental Aging Research. 1988;14:103-107.

25. Bopp KL, Verhaeghen P. Aging and verbal memory span: A metaanalysis. Journal of Gerontology: Psychological Sciences. 2005; 60B:223-233.

26. Feier CD, Gerstman LJ. Sentence comprehension abilities throughout the adult life span. Journal of Gerontology. 1980;35:722-728

27. Baum S. Sensitivity to syntactic violations across the age span: Evidence from a word monitoring task. Journal of Clinical Linguistics and Phonetics. 1991;5:317-328.

28. Waters GS, Caplan D. Age, working memory, and on-line syntactic processing in sentence comprehension. Psychology and Aging. 2001;16:128-144.

29. Davis GA, Ball HE. Effects of age on comprehension of complex sentences in adulthood. Journal of Speech and Hearing Research. 1989;32:143-150.

30. Obler LK, Fein D, Nicholas M, Albert, ML. Auditory comprehension and aging: Decline in syntactic processing. Applied Psycholinguistics. 1991;12:433-452.

31. Kemtes K, Kemper S. Younger and older adults' on-line processing of syntactically ambiguous sentence. Psychology and Aging. 1997;12:362-371.

32. Kemtes K. Decomposing adults' sentence comprehension: The role of age, working memory, inhibitory functioning, and perceptual speed. Unpublished doctoral dissertation, University of Kansas, Lawrence. 1999.

33. Van der Linden M, Hupet M, Feyereisen P, Schelstraete M, Bestgen Y, Bruyer MR, et al. Cognitive mediators of age-related differ- ences in language comprehension and verbal memory performance. Aging, Neuropsychology, and Cognition. 1999;6:11-24.

34. Stine-Morrow E, Ryan S, Leonard JS. Age differences in on-line syntactic processing. Experimental Aging Research. 2000;26:315322.

35. Kemper S, Sumner A. The structure of verbal abilities in older and younger adults. Psychology and Aging. 2001;16:312-322.

36. Hasher L, Zacks, RT. Working memory, comprehension, and aging: A review and new view. In G. H.Bower (Ed.), The psychology of learning and motivation. New York: Academic Press. 1988;193225.

37. Salthouse TA. The processing-speed theory of adult age differences in cognition. Psychological Review. 1996;103:403-428.

38. Novick JM, Trueswell JC, Thompson-Schill SL. Cognitive Control and Parsing: Reexamining the Role of Broca's Area in Sentence Comprehension. Cognitive, Affective, and Behavioral Neuroscience. 2005;5:263-281.

39. January D, Trueswell JC, Thompson-Schill SL. Co-localization of stroop and syntactic ambiguity resolution in Broca's area: implications for the neural basis of sentence processing. Cognitive Neuroscience. 2009;21:2434-2444.

40. Salthouse TA. Theoretical perspectives on cognitive aging. Hillsdale, NJ: Erlbaum; 1991.

41. Caplan D, DeDe G, Waters G, Michaud J, Tripodis Y. Effects of age, speed of processing and working memory on comprehension of sentences with relative clauses. Psychology and Aging. 2011;26(2): 439-450.

42. Bayles KA, Tomoeda CK. Arizona Battery for Communication disorders of Dementia (ABCD). Tucson, AZ: Canyonlands Publishing; 1993.

43. Rey A. L'examen psychologique dans le cas d'encephalopathie traumatique. Archives de Psycholgoie. 1941.p. 286-340.

44. Folstein M, Folstein SE, McHugh PR. "Mini-Mental State" a Practical Method for Grading the Cognitive State of Patients for the Clinician. Journal of Psychiatric Research. 1975;12(3):189-198.

45. Waters GS. Caplan D. The measurement of verbal working memory capacity and its relation to reading comprehension. Quarterly Journal of Experimental Psychology. 1996;49A:51-74.

46. Waters GS, Caplan D. The reliability and stability of operation span measures. Behavioral research Methods, Instruments \& Computers. 2003;35(4):550-564.

47. Eriksen BA, Eriksen CW. Effects of noise letters upon the identification of a target letter in a nonsearch task. Perception and Psychophysics, 1974;16:143-149.

48. Schneider W, Eschman A, Zuccolotto A. E-Prime reference guide. Pittsburgh, PA: Psychology Software Tools; 2002.

49. Muthén LK, Muthén BO. Mplus User's Guide. 5th ed. Los Angeles: Muthén \& Muthén, 2007.

50. Titone DA, Koh CK, Kjelgaard MM, Bruce S, Speer SR. Wingfield A. Age related impairments in the revision of syntactic misanalyses: Effects of prosody. Language and Speech. 2006;49(Pt1):75-99.

51. Efron B, Tibshirani RJ. An Introduction to the Bootstrap. London: Chapman \& Hall; 1993.

52. Waters GS, Caplan D. The relationship between age, processing 
speed, working memory capacity and language comprehension. Memory. 2005;13:403-413.

53. Ferreira F, Henderson JM. Reading processes during syntactic analysis and reanalysis. Canadian Journal of Experimental Psy- chology. 1993;47(2):247-275.

54. Schneiderman EI, Saddy JD. A linguistic deficit resulting from right hemisphere damage. Brain and Language. 1988;34(1):38-53. 